Jolyon Dales ${ }^{1}$, Pradeep Vasudevan² \& Marie-France Kong ${ }^{1}$

Department of Endocrinology, University Hospitals of Leicester NHS Trust, Leicester, UK Leicester Genetics Service, University Hospitals of Leicester NHS Trust, Leicester, UK

\section{Initial Presentation}

\section{Presentation "funny turns" \\ - Dizziness \\ - Lightheadedness \\ - Blurred vision \\ - Leg weakness}

-24-year-old traffic warden initially referred to the neurology department with episodes of

- Occurred over last seven years

- Resolve after sugary drink

- But relief not immediate

- Episodes could happen at any time of the day but never first thing in the morning and could happen several times a week.

-She reported that sometimes she gets spasms in her neck and arms, flopping of her head as well as weakness of her legs. -She had gained $10 \mathrm{~kg}$ over the past year.

\section{Other Medical History}

-Previous tonsillectomy

Initial Examination

-BP 128/53, no significant findings

\section{Further Information}

\section{Initial Family History}

\section{- Aunt has multiple sclerosis (initial FH} given in clinic)

- A MRI of her spine was normal.

\section{Progress}

- Symptoms attributed to hypoglycaemia and she was referred to the endocrinology unit for further investigations.

\section{Initial Investigations}

- Fasting blood glucose : $4.2 \mathrm{mmol} / \mathrm{L}$ $5.8 \mathrm{mmol} / \mathrm{L}$ on separate occasions

- Blood glucose during an attack was also normal

- Urea and electrolytes, thyroid function test and creatine kinase were normal

\section{Further Family History}

- On a subsequent clinic visit she was accompanied by her father who mentioned that the patient's mother was investigated for similar symptoms when she was 20 years old and several members of her family were also affected with similar symptoms.

\section{Hyperkalaemic Periodic Paralysis}

\section{- Hyperkalaemic periodic paralysis is a rare disorder, with an estimated prevalence of $1: 200,000$}

- Autosomal dominant inheritance with nearly complete penetrance.

- Point mutation in SCN4A gene on chromosome 17 leads to defective channel function

\section{Family History}

- Typically at least one first degree relative affected

- Family pedigree for the patient below
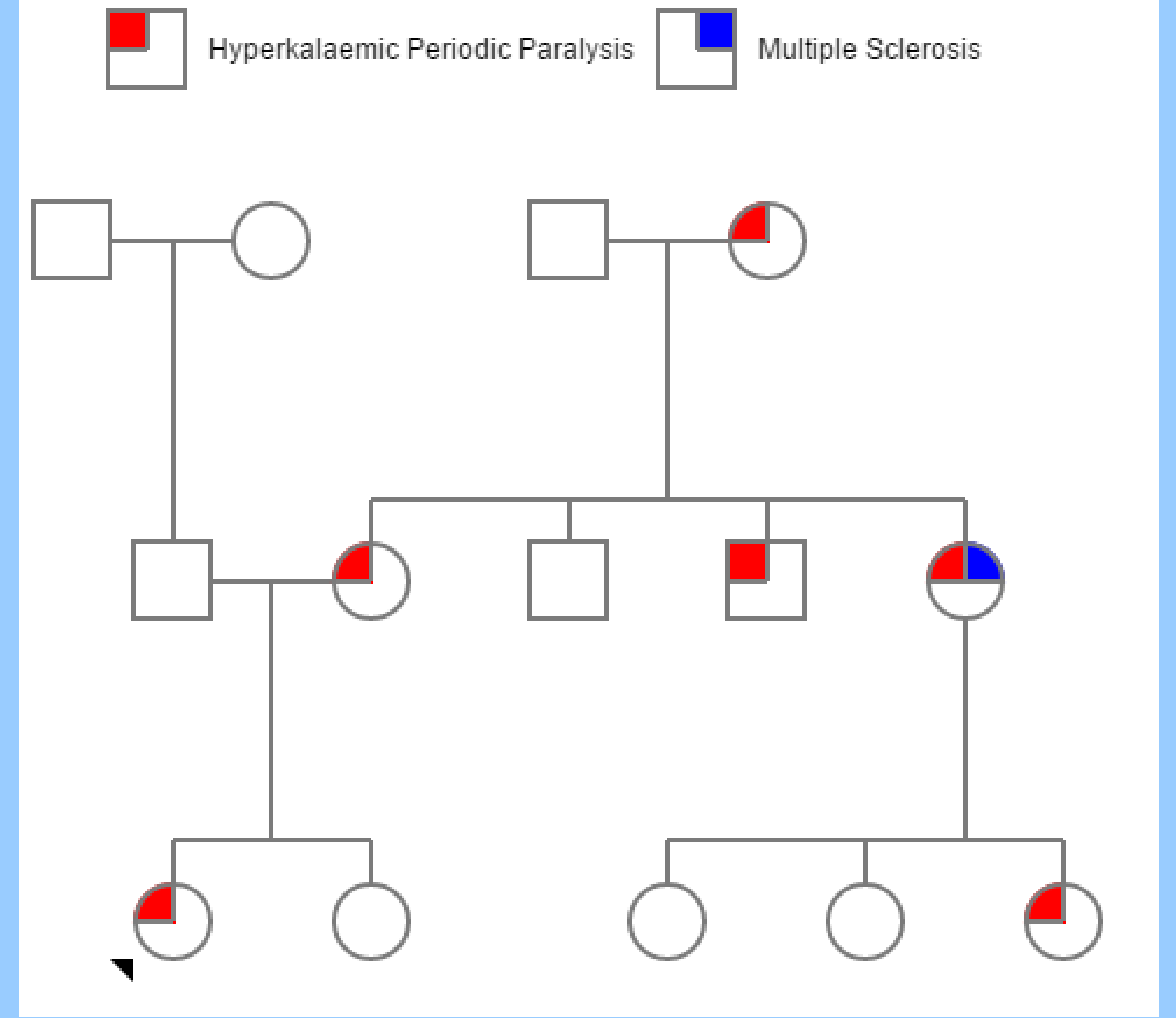

\section{Diagnosis}

Diagnosis should be suspected if

- At least two attacks of flaccid limb weakness

- Onset or worsening of attack with oral potassium intake

- Presents before age 20

- Absence of cardiac arrhythmias

- Normal Psychomotor development

$\underline{\text { Investigations }}$

\section{- During attacks}

- Hyperkalaemia - over $5 \mathrm{mmol} / \mathrm{L}$

-Rise of at least $1.5 \mathrm{mmol} / \mathrm{L}$ from baseline -Elevated creatine kinase (5-10x normal) -May see hyperkalaemic ECG changes

\section{- Between attacks}

- Normal serum potassium

-Normal muscle strength

-Mildly elevated creatine kinase
University Hospitals of Leicester WHS NHS Trust

\title{
Genetic Testing
}

\section{- Single Gene Testing \\ - Sequence analysis of SCN4A \\ - Multi-gene panel \\ - Includes SCN4A \\ - Other genes of interest \\ - Genetic testing of our patient is in progress at present}

\section{Management}

\section{Prevention}

- Avoiding Triggers

- Potassium rich foods

- Fasting

- Strenuous exercise

- Extreme cold

- Thiazide diuretics

Management of an attack

- Continuing mild exercise

- Carbohydrate intake (2g glucose/kg)

- Intravenous glucocorticoids

- 2 puffs salbutamol inhaler

- Intravenous calcium gluconate

\section{Summary}

Hyperkalaemic periodic paralysis is a rare cause of episodes of collapse

- History surrounding attacks can easily be mistaken for hypoglycaemia

-Establish family history and factors suggesting diagnosis prior to genetic testing

-Genetic testing can confirm diagnosis

-Distractors in family history can delay diagnosis

\section{References}

Koch MC, Ricker K, Otto M, et al. Confirmation of linkage of hyperkalaemic periodic paralysis to chromosome 17. Journal of Medical Genetics. 1991;28(9):583-586.

Weber F, Jurkat-Rott K, Lehmann-Horn F. Hyperkalemic Periodic Paralysis. In: Pagon RA, Adam MP, Ardinger HH, Wallace SE, Amemiya A, Bean LJH, et al., editors. GeneReviews(R). Seattle (WA): University of Washington, Seattle. University of Washington, Seattle. 1993.

Sansone VA, Burge J, McDermott MP, Smith PC, Herr B, Tawil $\mathbf{R}$, et al. Randomized, placebo-controlled trials of dichlorphenamide in periodic paralysis. Neurology. 2016;86(15):1408-16.

Charles G, Zheng C, Lehmann-Horn F, Jurkat-Rott K, Levitt J. Characterization of hyperkalemic periodic paralysis: a survey of genetically diagnosed individuals. Journal of neurology. 2013;260(10):2606-13. 